\title{
Correction: Resistance to anticancer vaccination effect is controlled by a cancer cell-autonomous phenotype that disrupts immunogenic phagocytic removal
}

\author{
Abhishek D. Garg ${ }^{1}$, Sanne Elsen ${ }^{2}$, Dmitri V. Krysko ${ }^{3,4}$, Peter Vandenabeele $^{3,4}$, Peter \\ de Witte ${ }^{2}$ and Patrizia Agostinis ${ }^{1}$ \\ ${ }^{1}$ Cell Death Research \& Therapy (CDRT) Unit, Department of Cellular and Molecular Medicine, KU Leuven University of \\ Leuven, Leuven, Belgium \\ ${ }^{2}$ Laboratory for Molecular Biodiscovery, Department of Pharmaceutical Sciences, KU Leuven, Leuven, Belgium \\ ${ }^{3}$ Molecular Signaling and Cell Death Unit, Department for Molecular Biomedical Research, VIB, Ghent, Belgium \\ ${ }^{4}$ Department of Biomedical Molecular Biology, Ghent University, Ghent, Belgium \\ Published: June 26, 2018
}

Copyright: Garg et al. This is an open-access article distributed under the terms of the Creative Commons Attribution License 3.0 (CC BY 3.0), which permits unrestricted use, distribution, and reproduction in any medium, provided the original author and source are credited.

This article has been corrected: The correct Supplementary Figure S2C is given below. The authors' explanation is as follows:

Due to erroneous figure processing and oversight, unintended mistakes occurred during the assembly of Supplementary Figure S2C - specifically, the BiP/Grp78, CHOP and Actin panels for the AY27-PDT samples only. Using the source/raw data, we have now generated the correct Supplementary Figure S2C.

The authors apologize for the oversight. The authors declare that this correction does not affect the description, interpretation, or the original conclusions of the manuscript.

c

CT26

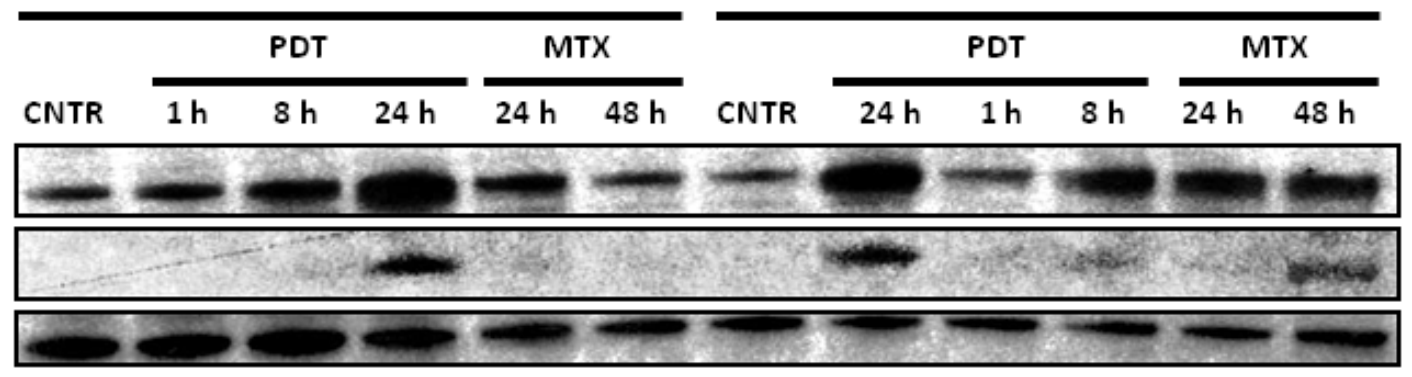

BiP/GRP78 (78 kDa)

CHOP (27 kDa)

$\beta-A c t i n(42 \mathrm{kDa})$

Supplementary Figure S2: Rat bladder carcinoma AY27 cells were treated or not (i.e. untreated controls/CNTR) with Hypericin-based Photodynamic Therapy (Hyp-PDT; $150 \mathrm{nM}$ Hyp incubated for $16 \mathrm{~h}$ followed by irradiation with light fluence of $2.70 \mathrm{~J} / \mathbf{c m}^{2}$ ) or mitoxantrone (MTX; $1 \mu \mathrm{M}$ ). C. immunoblotting for other ER stress markers like upregulation of BiP/GRP78 or CHOP levels at indicated recovery time-points. The calculations based on band densitometry analysis are mentioned as applicable.

Original article: Oncotarget. 2015; 6:26841-26860. https://doi.org/10.18632/oncotarget.4754 